\title{
Questionable alternative therapies for cancer, "The devil is in the detail”
}

\author{
Mohammad Nezami, MD* \\ Pacific Medical Center of Hope Fresno, CA, USA
}

\begin{abstract}
The wide usage of so called "alternative therapies" for cancer, has risen legitimate concerns over their safety and efficacy. This paper provides a summary of an anecdotal report intending to support the need for further studies before such therapies are commonly considered as neutral in the patent outcome, as some could negatively impact the treatment outcome and further patient survival. We conclude that further attention is required to ensure patients' safety is not over looked by prescribing alternative means of care by alternative practitioners.
\end{abstract}

\section{Background}

Cancer has been and is a prevalent disease with generally poor outcome. In 2019, there will be an estimated 1,762,450 new cancer cases diagnosed and 606,880 cancer deaths in the United States. In terms of cancer deaths between 2007 and 2016, the rate only decreased by $1.8 \%$ in men and $1.4 \%$ in women. The ten most commonly diagnosed cancers among men in the United States included cancers of the prostate, lung, colon, rectum, and bladder; melanomas of the skin; non-Hodgkins lymphoma; kidney cancer, mouth and throat cancer, leukemia, and pancreatic cancer. In women there has been a prevalence of 6,451,737 advanced cases reported by SEER at CDC and the most common cancers were reported as breast, lung and colon cancer. Every year, about 40 million Americans try alternative medicine. In the US, 42 prominent academic medical institutions including Cedars-Sinai, Harvard, Yale, Duke, and the Mayo clinic, are embracing integrative medicine modalities. That said, the application of such modalities are not formally being trained to the common practitioners [1].

Understanding the biology of the cancer cell and its relationship with the microenvironment recently has shown that the old concept of treating the person not the disease is in fact in concert with better survival. Also, there are significant advantage of some natural therapies in improving quality of life measures, especially in the areas of pain and psycho-emotional distress [2]. However, it is important for the patients and the providers to know that just by being natural, a compound or therapy is not necessarily safe or effective [3-6].

The range of so called "alternative therapies" can be from sham surgery to meditation, prayers, in one hand, and vigorously studied natural compounds and methods applied by scientific and well-studied merits on the other. In between there is a wide range of so called "claimed to be effective" therapies with significant invitro data but not enough information to support their use in human.

Although patients seek alternative therapies in the hope that it is less toxic, there are some concerns about variety of these methods, including lack of human studies/phase I trials to know the maximum tolerated dosing and the potential and harmful side effects and toxicity accompanied by their application [7-10].

\section{Methods}

In a series of 35 cases referred to our clinic, we retrospectedly reviewed the patients prior medical records specifically studying the intake of supplements and application of other alternative therapies, including blindly prescribed detoxification programs, high dose vitamins, antioxidants and enzymes. The results although not specific to one alternative method, showed progressive disease despite application of these modalities. We could not document any potential antineoplastic effects to support improved progression free survival as a result of these treatments. The progressive disease could also be caused by delay in receiving standard treatments as a result of replacement by alternative methods. Furthermore, the mechanism of action of many of these therapies, rely on antioxidant nature of these treatments and could well counteract the cellular response to alkylating agents used commonly as cytotoxic chemotherapy.

\section{Conclusion}

Although this study can be biased by the selection criteria, as the subjects of the study had referred to us for their care as a result of failure of prior therapies, the data is suggesting that non customized alternative therapies (that are promoted to replace standard treatments) could well fail to improve patients' survival which suggests that they are, at times, overused. That said, the quality of life measures were reported to be subjectively improved with the use of some of these alternative therapies, especially in advanced cancer. Further studies are required specifically to address the conflicts of action existing between specific standard treatments and each alternative mode of therapy.

\section{References}

1. National center of complementary and alternative health "The Use of Complementary and Alternative Medicine in the United States"

${ }^{*}$ Correspondence to: MA Nezami, MD, Pacific Medical Center of Hope Fresno, CA, USA, E-mail: amnezami@yahoo.com

Key words: alternative medicine, survival, cancer, quality of life

Received: February 02, 2019; Accepted: February 15, 2019; Published: February 18,2019 
2. Linda EC, Barry DB (2003) Benefits of psychosocial oncology care: Improved quality of life and medical cost offset. Health Qual Life Outcomes 1: 8. [Crossref]

3. Angell, M Kassirer JP (1998) Alternative medicine-the risks of untested and unregulated remedies. N Engl J Med 339: 839-841. [Crossref]

4. Coppes MJ, Anderson RA, Egeler RM (1998) Alternative therapies for the treatment of childhood cancer. $N$ Engl J Med 339: 846-847. [Crossref]

5. Chang EY, Glissmeyer M, Tonnes $\mathrm{S}$ (2006) Outcomes of breast cancer in patients who use alternative therapies as primary treatment. Am J Surg 192: 471-473. [Crossref]

6. Ernst E (2001) Intangible risks of complementary and alternative medicine. J Clin Oncol 19: 2365-2366. [Crossref]
7. Han E, Johnson N, DelaMelena T (2011) Alternative therapy used as primary treatment for breast cancer negatively impacts outcomes. Ann Surg Oncol 1: 912-916. [Crossref]

8. Joseph K, Vrouwe S Kamruzzaman A (2012) Outcome analysis of breast cancer patients who declined evidence-based treatment. World J Surg Oncol 10: 1. [Crossref]

9. Saqui J, Parker BA, Natarajan L (2012) Prognosis following the use of complementary and alternative medicine in women diagnosed with breast cancer. Complement Ther Med 20: 283-290. [Crossref]

10. Davis EL, Oh B, Butow PN (2012) Cancer patient disclosure and patient-doctor communication of complementary and alternative medicine use: A systematic review. Oncologist 17: 1475-1481. [Crossref]

Copyright: $\mathbb{C} 2019$ Nezami M. This is an open-access article distributed under the terms of the Creative Commons Attribution License, which permits unrestricted use, distribution, and reproduction in any medium, provided the original author and source are credited. 